\title{
Vegetation Database of Eurasian Tundra
}

\author{
Risto Virtanen
}

\begin{abstract}
The Vegetation Database of Eurasian Tundra (GIVD ID EU-FI-001) consists of vegetation plots from different parts of the Eurasian tundra. The sample plots represent high altitude vegetation on the northwestern Scandes both on siliceous and calcareous soil substrates, arctic tundra/polar semi-desert vegetation sampled along elevation and meso-topography gradients from 16 sites in total. The database is under development to include vegetation plots from tundra and mountain tundra vegetation published in the literature.
\end{abstract}

Keywords: arctic-alpine tundra; bryophyte; Finland; lichen; Spitsbergen; vascular plant.

GIVD Database ID: EU-FI-001

\section{Vegetation Database of Eurasian Tundra}

Scope: Vegetation plots from the mountain tundra areas the northern part of the Scandes, European arctic islands and tundra areas in Eurasia. Status: completed and continuing Period: $1986-1994$

Database manager(s): Risto Virtanen (risto.virtanen@oulu.fi)

Owner: Risto Virtanen, Botanical Museum, Univ. of Oulu (private)

Web address: http://www.oulu.fi/herboulu/english/index.html

Availability: according to a specific agreement

Online upload: no

Online search: no

Database format(s): Excel

Export format(s): Excel, CSV file, plain text file

Publication: Virtanen, R. \& Eurola, S. 1997. Middle oroarctic vegetation in Finland and middle-northern arctic vegetation on Svalbard. Acta Phytogeographica Suecica 82: 1-60.

Plot type(s): normal plots

Non-overlapping plots: 1,300

Total plot observations: 1,300

Plot-size range: $0.25-4 \mathrm{~m}^{2}$

Countries: $\mathrm{Fl}: 20.0 \%$; NO: $10.0 \%$; RU: $70.0 \%$

Estimate of existing plots: 2,500

Number of sources: 3

Completeness: $52 \%$

Valid taxa: 600

Forest: $0 \%$ - Non-forest: aquatic: $0 \%$; semi-aquatic: $0 \%$; arctic-alpine: $100 \%$; natural: $0 \%$; semi-natural: $0 \%$; anthropogenic: $0 \%$

Guilds: all vascular plants: 100\%; bryophytes (terricolous or aquatic): $100 \%$; lichens (terricolous or aquatic): $100 \%$

Environmental data: altitude: $100 \%$; slope aspect: $100 \%$; microrelief: $100 \%$; soil depth: $80 \%$; soil pH: $30 \%$

Performance measure(s): cover: $100 \%$

Geographic localisation: point coordinates less precise than GPS, up to $1 \mathrm{~km}: 100 \%$

Sampling periods: $1980-1989: 20.0 \% ; 1990-1999: 80.0 \%$

Information as of 2012-07-12; further details and future updates available from http://www.givd.info/ID/EU-FI-001

Risto Virtanen (risto.virtanen@oulu.fi)

Botanical Museum, University of Oulu, University of Oulu, 90014 Oulu, FINLAND 